\title{
fMRI for vegetative and minimally conscious states
}

\author{
A more balanced perspective
}

\author{
Lynne Turner-Stokes professor of rehabilitation ${ }^{1}$, Jenny Kitzinger professor of communications \\ research ${ }^{2}$, Helen Gill-Thwaites consultant occupational therapist ${ }^{3}$, E Diane Playford reader in \\ neurological rehabilitation ${ }^{4}$, Derick Wade professor of neurological rehabilitation ${ }^{5}$, Judith Allanson \\ consultant in neurorehabilitation ${ }^{6}$, John Pickard professor of neurosurgery ${ }^{7}$, On behalf of the Royal \\ College of Physicians' Prolonged Disorders of Consciousness Guidelines Development Group
}

\begin{abstract}
'King's College London School of Medicine, Department of Palliative Care, Policy and Rehabilitation, Cicely Saunders Institute, London SE5 9PJ, UK ; ${ }^{2}$ Cardiff University, School of Journalism, Media and Cultural Studies, UK; ${ }^{3}$ Royal Hospital for Neurodisability, London; ${ }^{4}$ Institute of Neurology, University College London, London; ${ }^{5}$ Nuffield Orthopaedic Centre, Oxford Centre for Enablement, Oxford, UK; ${ }^{6}$ Division of Neurosciences (Neurorehabilitation), Addenbrooke's Hospital, Cambridge, UK; ${ }^{7}$ Neurosurgery and Wolfson Brain Imaging Centre, University of Cambridge, Addenbrooke's Hospital
\end{abstract}

The BBC's Panorama programme The Mind Reader: Unlocking My Voice broadcast on 13 November 2012 provided important insights into the devastating experience of patients who live in vegetative or minimally conscious states and the families who support them. It also provided useful information on the use of functional magnetic resonance imaging (fMRI) to explore evidence of localised brain activity that might indicate underlying awareness. However, the programme failed to distinguish clearly between the two states and gave the impression that $20 \%$ of patients in a vegetative state show cognitive responses on fMRI. This claim needs to be clarified and put into perspective.

There are important differences between the two states. Patients in a vegetative state have no discernible awareness of self and no cognitive interaction with their environment. Patients in a minimally conscious state show evidence of interaction through localising or discriminating behaviours, although such interactions occur inconsistently. It is clinically important to make this distinction, for prognostic reasons and because some evidence suggests that patients in a minimally conscious state experience symptoms (such as pain) in a manner indistinguishable from non-brain injured patients. ${ }^{1}$

The programme presented two patients said to be in a "vegetative state" who showed evidence of cognitive interaction on assessment using fMRI in Ontario, Canada. The clinical methods used for the original diagnosis were not stated. In both cases, family members clearly reported that the patient made positive but inconsistent behavioural responses to questions. Within the programme, one of these patients was filmed responding to a question from his mother by raising his thumb and the other seemed to turn his head purposefully in response to having his earphones put on. These localising and discriminating features suggest that these patients were probably in a minimally conscious and not a vegetative state.

Studies of diagnostic accuracy show that more than $40 \%$ of patients in a minimally conscious state are misdiagnosed initially as being in a vegetative state. Systematic clinical evaluation of behaviours and responsiveness, assisted by structured assessments administered serially over time, may lead to a more accurate diagnosis. ${ }^{34}$ It is essential to exclude factors that may impede recovery, such as chronic hydrocephalus. Techniques for assessing disorders of consciousness vary between countries. ${ }^{5}$ In the United States, the Coma Recovery Scale-Revised (CRS-R) is widely used as a standardised assessment tool. ${ }^{6}$ In the United Kingdom, the Wessex Head Injury Matrix (WHIM) ${ }^{7}$ and the Sensory Modality Rehabilitation and Assessment Technique (SMART) ${ }^{8}$ which provides a more comprehensive evaluation of responses to five different sensory modalities, are more commonly used. Each tool can give slightly different results, and congruence between them requires further investigation.

Unsurprisingly, patients in a minimally conscious state often interact more readily with family and friends than they do with professionals. SMART-INFORMS is an important component of SMART that records the level of responses observed by family and friends. Video recordings of these interactions made by relatives may help the evaluation process by giving clinicians the opportunity to determine whether responses are truly localising and discriminating, or if they simply represent reflexive or spontaneous activity.

The Panorama programme also featured a patient who was diagnosed clinically as being in a vegetative state after prolonged multidisciplinary clinical evaluation (including SMART assessment) at the Royal Hospital for Neuro-disability in the 
UK. In his case, fMRI showed no evidence of cognitive interaction.

There are well documented cases where patients in a vegetative state, diagnosed according to current standards, have shown evidence of cortical responses on fMRI, ${ }^{9}$ but the $20 \%$ figure quoted in the programme is not supported by published evidence. In the largest published series (a convenience sample of 54 patients), ${ }^{10}$ one patient in a minimally conscious state and four in a vegetative state ( $9 \%$ in total) generated fMRI activity in response to motor or spatial imagery. The ability to respond to specific questions using this technique, however, has been reported in just one other case in the world literature. ${ }^{10}$

The possibility that fMRI might open up potential avenues of interaction for patients with profound communication deficits is an important finding, but the paradigms for testing and interpreting the findings are still to be determined. About one in five normal volunteers cannot generate fMRI activity on motor imagery tasks, so negative results in patients do not necessarily indicate lack of awareness.

fMRI is not suitable for all patients with reduced consciousness. Patients with metalwork, with frequent spontaneous movements, and those unable to lie flat are excluded. Alternative techniques for use at the bedside, or that require no active participation by the patient, are therefore being explored. ${ }^{11} 12$

Although the evidence so far is encouraging, it is still based on small numbers of highly selected patients. It is currently unclear whether fMRI can provide additional diagnostic information to that gained by careful and systematic behavioural assessment, or whether technological approaches have any prognostic use or could contribute to decision making in these patients. ${ }^{13}$ Currently, fMRI techniques are not sufficiently developed to form part of the standard assessment battery and should be applied only in the context of a registered national research programme. Imaging and other techniques must be accompanied by optimised clinical evaluation. This includes expert multidisciplinary assessment by appropriately experienced staff in specialist centres, conducted systematically using validated structured tools, and repeated over adequate periods of time. ${ }^{34}$

Guidelines for the management of patients in vegetative or minimally conscious states are being prepared by the Royal College of Physicians (due for publication in 2013). They will deal with the evidence base for different approaches to assessment in more detail and make recommendations for management through all stages of care, including sympathetic and responsible communication with patients' families and friends.

Competing interests: All authors have completed the ICMJE uniform disclosure form at www.icmje.org/coi_disclosure.pdf (available on request from the corresponding author) and declare: no support from any organisation for the submitted work; HG-T originally developed the SMART tool and receives some income as a SMART trainer; all authors have a clinical or academic interest in vegetative or minimally conscious states and have (or may at some time in the future) been involved in seeking or obtaining grant funding for research in this area; all authors except JK are employed by institutions that provide clinical services for patients in vegetative or minimally conscious states and sometimes provide expert testimony in this area; LT-S chairs the Royal College of Physicians working party for development of guidelines for prolonged disorders of consciousness and all co-authors are members of the working party; JK has a family member with profound brain injury. Provenance and peer review: Not commissioned; peer reviewed.

Luaute J, Maucort-Boulch D, Tell L, Quelard F, Sarraf T, Iwaz J, et al. Long-term outcomes of chronic minimally conscious and vegetative states. Neurology 2010;75:246-52.

2 Boly M, Faymonville ME, Schnakers C, Peigneux P, Lambermont B, Phillips C, et al. Perception of pain in the minimally conscious state with PET activation: an observational study. Lancet Neurol 2008;7:1013-20.

3 Gill-Thwaites H. Lotteries, loopholes and luck: misdiagnosis in the vegetative state patient. Brain Inj 2006;20:1321-8.

4 Schnakers C, Vanhaudenhuyse A, Giacino J, Ventura M, Boly M, Majerus S, et al. Diagnostic accuracy of the vegetative and minimally conscious state: clinical consensus versus standardized neurobehavioral assessment. BMC Neurol 2009;9:35.

5 Seel RT, Sherer M, Whyte J, Katz DI, Giacino JT, Rosenbaum AM, et al. Assessment scales for disorders of consciousness: evidence-based recommendations for clinical practice and research. Arch Phys Med Rehabil 2010;91:1795-813.

6 Giacino JT, Kalmar K, Whyte J. The JFK Coma Recovery Scale-Revised: measurement characteristics and diagnostic utility. Arch Phys Med Rehabil 2004;85:2020-9.

7 Shiel A, Horn SA, Wilson BA, Watson MJ, Campbell MJ, Mclellan DL. The Wessex Head Injury Matrix (WHIM) main scale: a preliminary report on a scale to assess and monitor patient recovery after severe head injury. Clin Rehabil 2000:14:408-16.

8 Gill-Thwaites $\mathrm{H}$. The Sensory Modality Assessment Rehabilitation Technique-a tool for assessment and treatment of patients with severe brain injury in a vegetative state. Brain Inj 1997;11:723-34.

9 Owen AM, Coleman MR, Boly M, Davis MH, Laureys S, Pickard JD. Detecting awareness in the vegetative state. Science 2006;313:1402.

10 Monti MM, Vanhaudenhuyse A, Coleman MR, Boly M, Pickard JD, Tshibanda L, et al. Willful modulation of brain activity in disorders of consciousness. N Engl J Med 2010;362:579-89.

11 Fernandez-Espejo D, Bekinschtein T, Monti MM, Pickard JD, Junque C, Coleman MR, et al. Diffusion weighted imaging distinguishes the vegetative state from the minimally conscious state. Neuroimage 2010;54:103-12.

12 Fernandez-Espejo D, Soddu A, Cruse D, Palacios EM, Junque C, Vanhaudenhuyse A, et al. A role for the default mode network in the bases of disorders of consciousness. Ann Neurol 2012;72:335-43.

13 Coleman MR, Davis MH, Rodd JM, Robson T, Ali A, Owen AM, et al. Towards the routine use of brain imaging to aid the clinical diagnosis of disorders of consciousness. Brain 2009;132:2541-52

Cite this as: $B M J$ 2012;345:e8045

(c) BMJ Publishing Group Ltd 2012 